\title{
Diabetes mellitus in pregnancy does not delay postpartum pelvic floor recovery
}

\author{
NETA BENSHALOM-TIROSH, (1) MIRIAM ERENBERG, () YAEL BAUMFELD, (1) ZEHAVA YOHAY, (1) HANNAH GLINTER, \\ (1) ELLA PARDO, (1) DAN TIROSH, (1) DAVID YOHAY, (1) ADI Y. WEINTRAUB
}

Department of Obstetrics and Gynaecology, Soroka University Medical Center and Faculty of Health Sciences, Ben-Gurion University of the Negev, BeerSheva, Israel

\section{ABSTRACT}

Objective: Evaluate the impact of diabetes mellitus (DM) on postpartum pelvic floor dysfunction symptoms recovery.

Materials and Methods: A prospective cross-sectional study. Participants completed "Pelvic Floor Distress Inventory-20" questionnaires during third trimester, and three months postpartum. Statistical analysis was conducted to determine differences between questionnaire scores in both periods, comparing recovery among women with and without diabetes in pregnancy.

Results: A total of 192 participants were divided into diabetes $(n=66)$ and control $(n=126)$ groups. At three months postpartum, 114/192 (59.4\%) women completed both questionnaires. There was a significant difference between the questionnaire summary score during pregnancy, and three months postpartum $(p<0.001)$. No significant differences were noted in the extent of symptoms recovery between women with and without diabetes ( $\mathrm{p}=0.2$ for total score).

Conclusion: There is a clinically and statistically significant spontaneous recovery of pelvic floor dysfunction symptoms in the postpartum period. DM in pregnancy does not delay this postpartum recovery.

Keywords: Colorectal anal dysfunction; diabetes mellitus; pelvic floor dysfunction; PFDI-20; urinary incontinence

\section{INTRODUCTION}

Pelvic floor disorders (PFD) include pelvic organ prolapse (POP), colorectal and anal dysfunction (CRAD) and urinary distress (UD). PFD symptoms have a major effect on women's quality of life, with psychosocial effects including discomfort, anxiety, embarrassment, loss of self-esteem and frustration. ${ }^{1}$ The treatment of PFD holds an overwhelming economic burden, and is a major public health concern. ${ }^{2}$
The reported prevalence of PFD during and after pregnancy varies. $^{3}$ Many risk factors are involved in pregnancy and postpartum PFD, with growing evidence for the important impact of the mode of delivery. ${ }^{4}$ The effects of pregnancy, even without birth-related pelvic floor injury, have also been implicated as an independent risk factor for PFD. ${ }^{5}$ Other pregnancy related risk factors investigated include parity, instrumental delivery, obstetrical trauma, episiotomy, length of the first and second stages of labor, use of epidural anesthesia and neonatal weight. ${ }^{6}$ 
However, many aspects of the PFD pathophysiology associated with pregnancy, delivery and the puerperium remain unknown.

The prevalence of diabetes mellitus (DM) during pregnancy is about $7 \%$, most cases being gestational DM. ${ }^{7}$ Of note, there is a constant increase of the prevalence of DM due to the gradual increase in Body Mass Index and the epidemic of obesity during recent decades. ${ }^{8}$ Diabetes in pregnancy, both gestational diabetes mellitus (GDM) and pregestational DM, is an independent risk factor for obstetrical complications affecting both the mother and the fetus, including gestational hypertension, a higher rate of cesarean deliveries, large for gestational age neonates, and shoulder dystocia. ${ }^{9}$

Diabetes and pre-diabetic conditions, such as insulin resistance and impaired fasting glucose, have been described as risk factors for PFD, especially for urinary incontinence. ${ }^{10}$ However, there is scarce data regarding the relationship of DM during pregnancy to PFD symptoms. ${ }^{11}$ This relationship might be of interest, since gestational DM and insulin resistance during pregnancy are often transient conditions, coming to resolution after the early postpartum period.

The Pelvic Floor Distress Inventory-20 (PFDI-20) questionnaire is a short version of the Pelvic Floor Distress Inventory (PFDI), ${ }^{12}$ designed to assess the extent of PFD symptoms and their effect on the patient's quality of life. ${ }^{13}$ This questionnaire has been validated for use in the Hebrew language as well as for the pregnant population. ${ }^{14,15}$

The aim of this study was to investigate the prevalence of PFD symptoms in Israeli women with and without DM in pregnancy. Furthermore, we intended to compare the postpartum recovery from PFD symptoms in these women and evaluate various obstetrical factors that may correlate with these changes.

\section{MATERIALS AND METHODS}

A prospective cross-sectional study was conducted between February 2017 to June 2017 in the Department of Obstetrics and Gynaecology at the Soroka University Medical Center (SUMC), Beer Sheva, Israel. SUMC is a tertiary center serving a population of more than a million, with approximately 17,000 deliveries annually. Approval of the Institutional Review Board was obtained; verbal informed consent was given prior to administrating the questionnaires.

PFDI-20 in the Hebrew language was distributed to all participants. The PFDI-20 consists of items that concern the three components of PFD: ${ }^{16}$ 1) POP distress inventory (POPDI), 6 items; 2) colorectal and anal dysfunction (CRAD) inventory, 8 items; and 3) urinary distress inventory (UDI), 6 items. Participants are requested to answer either yes or no to the questionnaire items. "No" is given a value of "0" whereas the answer "yes" is followed by a scale of bother, ranking between 1 and 4 ( $1=$ "not at all"; 2 = "somewhat"; 3 = "moderately"; and 4 = "quite a bit"). For each patient, there is an option to calculate a Scale Score and a Summary Score. The Scale Score is the mean value of all questions answered per scale multiplied by 25 , so that each scale (POP, CRAD and UD), may receive a maximum score of 100. The Summary Score is the sum of all three Scale Scores (range: $0-300)$.

Inclusion criteria included age over 18 years, delivery at SUMC and adequate understanding of the Hebrew language. Inclusion criteria for the diabetes group included women with either a pregestational diagnosis of DM, or diagnosed with GDM during the index pregnancy, based on an oral glucose tolerance test, or fasting glucose indicating overt diabetes. ${ }^{17}$ Exclusion criteria included multiple pregnancies.

Patients were recruited either during the third trimester of pregnancy at the outpatient clinic, or at labor and delivery or maternity ward within 24 hours of delivery. After giving an informed consent, women were asked to fill out the PFDI-20 questionnaire (reflecting symptoms in the third trimester), either by filling the questionnaire form or assisted by an interviewer. All women gave their consent for a follow-up questionnaire three months after delivery, to reflect the women's state during the postpartum period. The second questionnaire was sent via an electronic application, and women who did not respond electronically were contacted for a telephone interview.

Data regarding maternal baseline characteristics, including age, gravidity, parity as well as clinical information regarding the index delivery (e.g. mode of delivery, neonatal birth weight, perineal tears), were obtained from the hospital's computerized medical records.

\section{Statistical analysis}

Statistical analysis was performed using the SPSS software package, version 20 (SPSS Inc, Chicago, IL). Categorical variable data is presented using percentile and statistical significance was tested using the $X^{2}$ or Fisher's exact test, as appropriate. Numerical variable data is presented using median and interquartile range and statistical significance was analyzed using Mann-Whitney $\mathrm{U}$ test. Continuous variable data is presented using mean and standard deviation, Student t-test was used for statistical analysis, paired t-test was used when appropriate. P-value of 0.05 and under was considered statistically significant.

A post-hoc sample size calculation was performed using the data found in our study. Considering a ratio of 1:1 in the control versus the study group and a mean total score for the study 
group of 70 with SD of 47 and for the control of 28 with SD of 37 , the sample size calculated with a power of $80 \%$ and alpha of 0.05 was 20 in each group.

\section{RESULTS}

A total of 192 women were recruited during the study period, 66 women in the DM group and 126 in the control group. At three months postpartum, 114/192 (59.4\%) women have completed the second questionnaire, and were included in the analysis comparing both periods. Of these, $45 / 66$ (68.2\%) women in the DM group and 69/126 (54.8\%) in the control group. Baseline characteristics of both groups are presented in Table 1. Maternal age and maternal weight were significantly higher among the DM group. Perinatal and neonatal characteristics are presented in Table 1. Women with DM during pregnancy delivered earlier than the control group (38.5 \pm 1.3 weeks vs $39.2 \pm 2.2$ weeks of gestation, respectively, $p=0.02$ ), and had a larger proportion of

\begin{tabular}{|c|c|c|c|c|}
\hline \multicolumn{5}{|c|}{$\begin{array}{l}\text { Table 1. Demographic, perinatal and neonatal characteristics } \\
\text { of the diabetes and the control groups }\end{array}$} \\
\hline \multicolumn{2}{|c|}{ Variables } & $\begin{array}{l}\text { Diabetes } \\
(n=66)\end{array}$ & $\begin{array}{l}\text { No diabetes } \\
(n=126)\end{array}$ & $p$-value \\
\hline \multicolumn{2}{|c|}{ Maternal age, (year) } & $32.3 \pm 5.2$ & $29.3 \pm 4.9$ & $<0.001$ \\
\hline \multicolumn{2}{|c|}{ Weight, (kg) } & $85.4 \pm 20$ & $76.7 \pm 14.9$ & 0.002 \\
\hline \multicolumn{2}{|c|}{ Height, (cm) } & $162.3 \pm 7.1$ & $163.1 \pm 6.7$ & 0.63 \\
\hline \multicolumn{2}{|c|}{ Gravidity } & $3.24 \pm 1.94$ & $2.86 \pm 1.84$ & 0.17 \\
\hline \multicolumn{2}{|l|}{ Parity } & $1.59 \pm 1.67$ & $1.42 \pm 1.34$ & 0.68 \\
\hline \multicolumn{2}{|c|}{ Previous vaginal deliveries } & $1.38 \pm 1.72$ & $1.23 \pm 1.40$ & 0.93 \\
\hline \multicolumn{2}{|c|}{ Previous cesarean sections } & $0.21 \pm 0.57$ & $0.10 \pm 0.35$ & 0.18 \\
\hline \multicolumn{2}{|c|}{ Past miscarriages } & $0.65 \pm 0.95$ & $0.44 \pm 0.72$ & 0.22 \\
\hline \multicolumn{2}{|c|}{ Delivery week, (weeks) } & $38.5 \pm 1.3$ & $39.2 \pm 2.2$ & 0.02 \\
\hline \multicolumn{2}{|c|}{ Birth weight, (grams) } & $3,221 \pm 471$ & $3,233 \pm 553$ & 0.89 \\
\hline \multicolumn{2}{|c|}{$\begin{array}{l}\text { Duration of } 2^{\text {nd }} \text { stage, } \\
\text { (minutes) }\end{array}$} & $47.4 \pm 81.5$ & $51.1 \pm 74.2$ & 0.79 \\
\hline \multicolumn{2}{|c|}{$\begin{array}{l}\text { Duration of } 3^{\text {rd }} \text { stage, } \\
\text { (minutes) }\end{array}$} & $12.8 \pm 8.4$ & $12.5 \pm 5.3$ & 0.82 \\
\hline \multicolumn{2}{|c|}{$\begin{array}{l}\text { Time from rupture of } \\
\text { membranes to delivery, } \\
\text { (minutes) }\end{array}$} & $338 \pm 341$ & $357 \pm 411$ & 0.82 \\
\hline \multirow{3}{*}{$\begin{array}{l}\text { Mode of } \\
\text { delivery }\end{array}$} & Vaginal & $43(66.2 \%)$ & $101(80.2 \%)$ & 0.01 \\
\hline & Vacuum & $2(3.1 \%)$ & $8(6.3 \%)$ & \\
\hline & CS & $20(30.8 \%)$ & $17(13.5 \%)$ & \\
\hline \multicolumn{2}{|c|}{ Epidural anestesia } & $27(41.5 \%)$ & $42(33.6 \%)$ & 0.19 \\
\hline \multicolumn{2}{|c|}{ Episiotomy } & $1(1.5 \%)$ & $11(8.7 \%)$ & 0.06 \\
\hline \multicolumn{2}{|c|}{$3^{\text {rd }}$ degree tear } & $0(0 \%)$ & $2(1.6 \%)$ & NS \\
\hline \multicolumn{2}{|c|}{$4^{\text {th }}$ degree tear } & $1(1.6 \%)$ & $0(0 \%)$ & NS \\
\hline
\end{tabular}

cesarean deliveries ( $30.8 \%$ vs $13.5 \%, p=0.01)$. No differences were noted between the groups regarding the mean birthweight, rates of epidural anesthesia, the duration of second stage of labor and perineal tears.

The decrease in self-reported PFD symptoms three months postpartum compared with during pregnancy was statistically significant in 12/20 PFDI items, in our study population (Table 2). The overall PFDI-20 score was significantly decreased $(p<0.001)$, and this decrease was significant for all components of the PFDI20: POP distress $(p<0.001)$; colorectal and anal distress $(p=0.01)$; and UD $(p<0.001)$.

The differences in the mean PFDI-20 scores at third trimester versus three months postpartum (delta score change) among patients with and without diabetes are presented in Table 3 and Figure 1. No significant differences were noted in the extent of

\section{Table 2. Differences in PFDI-20 Scores during pregnancy} and three months postpartum among the study participants

\begin{tabular}{|l|l|l|l|}
\hline Variables & $\begin{array}{l}\text { During } \\
\text { pregnancy }\end{array}$ & $\begin{array}{l}\text { Three months } \\
\text { postpartum }\end{array}$ & p-value \\
\hline PFDI-1 & $1.82 \pm 1.36$ & $0.26 \pm 0.79$ & $<0.001$ \\
\hline PFDI-2 & $1.42 \pm 1.51$ & $0.30 \pm 0.78$ & $<0.001$ \\
\hline PFDI-3 & $0.39 \pm 0.95$ & $0.17 \pm 0.71$ & 0.04 \\
\hline PFDI-4 & $0.38 \pm 1.02$ & $0.31 \pm 0.96$ & 0.71 \\
\hline PFDI-5 & $1.30 \pm 1.49$ & $0.31 \pm 0.74$ & $<0.001$ \\
\hline PFDI-6 & $0.06 \pm 0.33$ & $0.03 \pm 0.29$ & 0.55 \\
\hline PFDI-7 & $0.82 \pm 1.32$ & $0.46 \pm 1.28$ & 0.14 \\
\hline PFDI-8 & $0.80 \pm 1.24$ & $0.48 \pm 0.99$ & 0.03 \\
\hline PFDI-9 & $0.11 \pm 0.57$ & $0.08 \pm 0.46$ & 0.71 \\
\hline PFDI-10 & $0.19 \pm 0.70$ & $0.09 \pm 0.49$ & 0.17 \\
\hline PFDI-11 & $0.74 \pm 1.24$ & $0.66 \pm 1.25$ & 0.81 \\
\hline PFDI-12 & $0.77 \pm 1.26$ & $0.54 \pm 1.17$ & 0.07 \\
\hline PFDI-13 & $1.06 \pm 1.50$ & $0.68 \pm 1.17$ & 0.01 \\
\hline PFDI-14 & $0.50 \pm 1.23$ & $0.30 \pm 0.87$ & 0.33 \\
\hline PFDI-15 & $2.32 \pm 1.42$ & $0.65 \pm 1.12$ & $<0.001$ \\
\hline PFDI-16 & $1.02 \pm 1.47$ & $0.40 \pm 0.94$ & $<0.001$ \\
\hline PFDI-17 & $1.39 \pm 1.55$ & $0.75 \pm 1.29$ & $<0.001$ \\
\hline PFDI-18 & $1.13 \pm 1.47$ & $0.52 \pm 1.11$ & $<0.001$ \\
\hline PFDI-19 & $0.56 \pm 1.13$ & $0.11 \pm 0.50$ & $<0.001$ \\
\hline PFDI-20 & $1.32 \pm 1.49$ & $0.30 \pm 0.85$ & $<0.001$ \\
\hline Scale Score POP & $22.31 \pm 17.01$ & $5.71 \pm 11.19$ & $<0.001$ \\
\hline Scale Score CRAD & $15.54 \pm 16.31$ & $10.31 \pm 15.46$ & 0.01 \\
\hline Scale Score UDI & $32.25 \pm 22.73$ & $11.33 \pm 16.40$ & $<0.001$ \\
\hline Summary score & $70.11 \pm 46.64$ & $27.57 \pm 36.57$ & $<0.001$ \\
\hline PFDI: Pelvic Floor Disability Index; POP: Pelvic organ prolapse; CRAD: \\
deviation \\
Data is presented as mean \pm SD & & Standard \\
\hline & & & \\
\hline
\end{tabular}




\begin{tabular}{|l|l|l|l|}
\hline \multicolumn{4}{|l|}{$\begin{array}{l}\text { Table 3. The Change in Pelvic Floor Dysfunction Score during } \\
\text { pregnancy and three months postpartum }\end{array}$} \\
\hline Variables & $\begin{array}{l}\text { Diabetes } \\
\text { (n=45) }\end{array}$ & $\begin{array}{l}\text { No diabetes } \\
\text { (n=69) }\end{array}$ & p-value \\
\hline $\begin{array}{l}\text { POP Score - during } \\
\text { pregnancy }\end{array}$ & $19.07 \pm 17.95$ & $20.17 \pm 15.18$ & 0.73 \\
\hline $\begin{array}{l}\text { POP Score - 3 } \\
\text { months postpartum }\end{array}$ & $7.13 \pm 12.13$ & $5.12 \pm 10.81$ & 0.36 \\
\hline Delta POP & $-13.70 \pm 17.92$ & $-15.53 \pm 15.83$ & 0.57 \\
\hline $\begin{array}{l}\text { CRAD Score - during } \\
\text { pregnancy }\end{array}$ & $14.17 \pm 13.95$ & $13.99 \pm 13.74$ & 0.95 \\
\hline $\begin{array}{l}\text { CRAD Score - 3 } \\
\text { months postpartum }\end{array}$ & $13.14 \pm 19.30$ & $8.65 \pm 12.72$ & 0.18 \\
\hline Delta CRAD & $-1.34 \pm 15.03$ & $-5.34 \pm 14.43$ & 0.16 \\
\hline $\begin{array}{l}\text { UDI Score - during } \\
\text { pregnancy }\end{array}$ & $31.85 \pm 23.82$ & $28.68 \pm 23.15$ & 0.48 \\
\hline $\begin{array}{l}\text { UDI Score - 3 months } \\
\text { postpartum }\end{array}$ & $15.00 \pm 19.00$ & $9.42 \pm 14.57$ & 0.10 \\
\hline Delta UDI & $-16.85 \pm 20.45$ & $-19.26 \pm 19.43$ & 0.53 \\
\hline $\begin{array}{l}\text { Total score - during } \\
\text { pregnancy }\end{array}$ & $65.09 \pm 46.53$ & $62.85 \pm 42.98$ & 0.79 \\
\hline $\begin{array}{l}\text { Total score - 3 } \\
\text { months postpartum }\end{array}$ & $34.98 \pm 42.83$ & $23.65 \pm 32.20$ & 0.13 \\
\hline Delta total score & $-30.11 \pm 37.10$ & $-39.38 \pm 37.10$ & 0.20 \\
\hline $\begin{array}{l}\text { POP: Pelvic organ prolapse; CRAD: Colorectal-anal distress; UDI: Urinary } \\
\text { distress inventory; } \mathrm{n} \text { Number } \\
\text { Data is presented as mean } \pm \text { SD }\end{array}$ & & \\
\hline
\end{tabular}

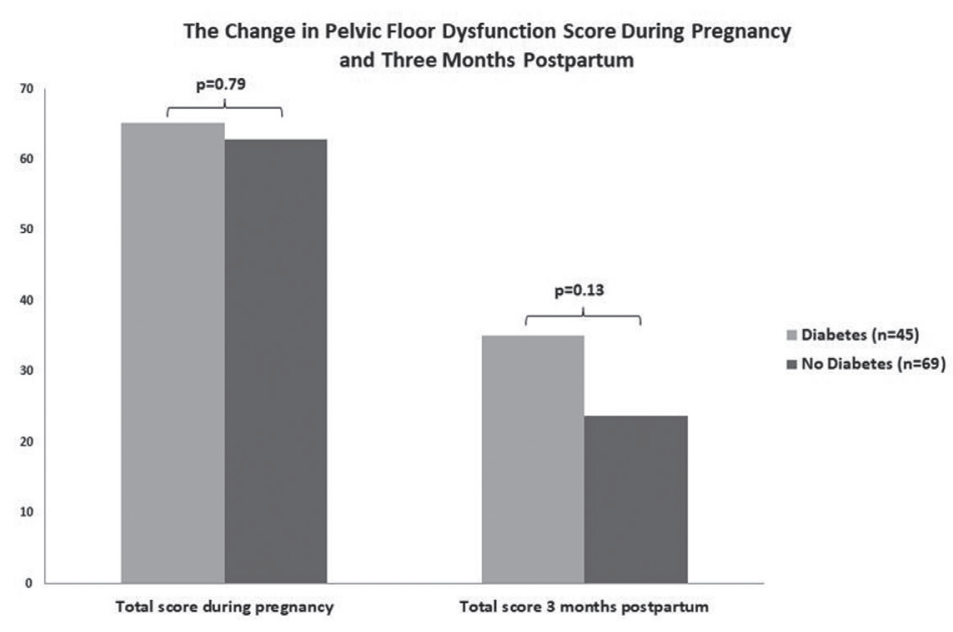

Figure 1. The change in Pelvic Floor Dysfunction Score during pregnancy and three months postpartum

recovery of the overall PFD symptoms between women with DM and the control group $(p=0.2)$. This was also the case when stratifying these changes according to the three components of PFDI-20 [delta POP score $(p=0.57)$, delta CRAD score $(p=0.16)$ and delta UDI score $(p=0.53)]$.

\section{DISCUSSION}

Pelvic floor symptoms are a common complaint during pregnancy, specifically during the third trimester and the immediate postpartum period. These symptoms can be reversed during the puerperium, although they have been reported to sustain as long as 6-12 months after delivery. ${ }^{18}$ Parity and pregnancy outcomes, such as mode of delivery and labor dystocia as well as perineal tears, ${ }^{6,15}$ have been recognized as risk factors for long term PFD. Women still experiencing PFD symptoms postpartum reported a negative effect on health-related quality of life measures. ${ }^{19}$

The effect of pregnancy on PFD symptoms is thought to result from the influence of the growing uterus and increased weight bearing of the pelvic floor muscles, as well as from hormonal changes. ${ }^{20}$ During pregnancy, collagen rods begin to loosen in a response to secretion of placental hormones, specifically relaxin, ${ }^{21}$ resulting in weakened ligaments. This process accelerates $24-48$ hours before labor, allowing the ligaments to stretch against the fetus' head during delivery. These changes contribute to the prevalence of PFD during the second and third trimester. ${ }^{21}$ However, the postpartum resolution of the mechanical and hormonal changes does not explain the persistence of symptoms over an extended period of time. A possible explanation is that PFD symptoms may develop postpartum in some patients, thus maintaining an overall similar prevalence of these complaints. ${ }^{5,11}$ Additionally, since PFD symptoms have even been previously reported to be sustained as long as 6-12 months after delivery, it is possible that the three months follow up period undertaken in many studies may represent an uncomplete recovery.

Previous reports have established an association between DM and pelvic floor symptoms. The prevalence of urinary stress incontinence was found to be 33\%-39\% in women with impaired fasting glucose or diabetes, compared to $16 \%-26 \%$ in the control populations. ${ }^{10,22}$ This may be partially related to the association between DM and obesity; although both have been shown to be independent risk factors for PFD. ${ }^{23}$ Possible explanations for this association between urinary incontinence and other PFD symptoms to DM have been related to microvascular changes and peripheral neuropathy, in addition to polyuria symptoms associated with diabetes. ${ }^{24}$ Additional factors impairing adequate tissue repair and wound healing may also play a role in the development of PFD in diabetic patients (for example POP). ${ }^{25}$ There is also evidence that DM and insulin resistance alter the electromyographic activity of the pelvic floor muscles. ${ }^{26}$

In the current study, there was an overall improvement in PFD scores in both the DM and the control groups three months postpartum. In a previous study, comparing PFDI scores during the third trimester and three months postpartum in 
our population, in almost half (9/20) of the PFDI items there was a significant change between the two study periods, with mixed trends of improvement or worsening. ${ }^{15}$ In the current study, 12/20 items were significantly different between the two study periods, and all three components of the PFDI showed statistically significant improved scores.

A limited number of studies have specifically investigated the association between GDM and the postpartum recovery of pelvic floor symptoms. Chung et al. ${ }^{11}$ evaluated in a large cohort of over 6,000 women the effect of GDM on urinary incontinence during pregnancy and a postpartum follow-up and found that GDM was an independent risk factor for urinary incontinence, and that women continued to exhibit urinary stress incontinence symptoms up to two years postpartum, although there was no reference to the effect of GDM on the recovery rate. ${ }^{11}$ Of note, women in the GDM group had a higher rate of neonates with a birthweight $\geq 4000$ gr ( $9 \%$ compared with $2.7 \%$ for non GDM), but also a higher rate of cesarean delivery $(46.7 \%$ vs $32.2 \%$ in non GDM group), which in turn offers potential benefit in terms of pelvic floor injury. ${ }^{11}$ Kim et al. ${ }^{27}$ surveyed women up to 5 years from delivery and analyzed the frequency of urinary incontinence during and after a pregnancy with GDM. Around $50 \%$ of their population reported incontinence symptoms at least once a week during and after pregnancy. Surprisingly, the rate of women reporting symptoms of incontinence was significantly higher as the interval from delivery increased. This may be due to age, or to other variables affected by the latency period from delivery to survey. ${ }^{27}$

Our prospective analysis has shown no statistically significant difference in PFD symptoms between patients with and without DM in pregnancy. Moreover, no effect on the postpartum recovery of PFD symptoms as evident from the PFDI scores was noted between patients with or without DM in pregnancy. To the best of our knowledge this has not been directly assessed in previous studies. Our findings imply that the postpartum improvement in PFD symptoms is probably related to the resolution of the mechanical and hormonal changes affecting these symptoms during pregnancy, rather than diabetes associated changes. However, future studies should focus on the long-term effect of DM in pregnancy on PFD.

The strengths of this study are its prospective design as well as the use of validated questionnaires to assess pelvic floor symptoms. Our population is a heterogeneous population of diverse ethnicity and parity which increases the generalizability of these findings to the Israeli population as well as to other countries.

One of the major limitations of the study is the heterogeneity of the DM group which included both GDM ( $n=48$, of which
28 did not require neither oral nor insulin treatment) and pregestational DM $(n=9)$, as well as women with DM with unknown time of onset $(n=9)$. This heterogeneity, as well as heterogeneity in recorded treatment for DM (oral hypoglycemic treatment in $\mathrm{n}=10$, insulin treatment in $\mathrm{n}=20$, the rest not recorded) has possibly impaired the ability to isolate the effect of diabetes during the gestational period on PFD symptoms. However, if pregestational diabetes, having a greater potential for longterm diabetic complications, has indeed affected the results, the fact that the postpartum PFDI scores for the diabetes group were still not significantly different from the non-diabetic controls, strengthens our argument that diabetes was not a significant factor in postpartum PFD symptoms recovery. Another consideration is that the population of the study was a relatively young population, with a mean maternal age of 32 in the diabetes group, and therefore the plausibility that these women who have pregestational diabetes suffer from advanced microvascular disease is small.

A second limitation is that the response rate for the second questionnaire was about $60 \%$. It is noteworthy that this response rate was higher compared to previous studies in our population, especially in the DM group. ${ }^{15}$ The higher response rate in the current study may be attributed to the fact that for the second questionnaire women were first approached electronically, and those who did not respond were contacted by a telephone interview.

Patients were recruited either during the third trimester of pregnancy at the outpatient clinic, at labor and delivery or the maternity ward within 24 hours of delivery. It is possible that the first 24 hours after delivery is not the optimal timing for recruitment, as some women were too weak or did not feel up to participating in the study after delivery.

Differences in mode of delivery may have also served as a limitation in the current study, as the vaginal delivery rate was higher among non-diabetic group, possibly leading to more extensive vaginal floor injury, or delayed recovery, minimizing potential differences between diabetic and non-diabetic groups. Finally, there is a possible bias due to the difference between methods of answering both questionnaires (first; self-reported questionnaire or interview, second; electronic version or telephone interview). Women who first responded by selfreporting may feel reluctant to answer personal questions over the phone, thus "minimizing" the report of their discomfort.

\section{CONCLUSION}

Our study has demonstrated that there is a clinically and statistically significant spontaneous recovery from PFD 
symptoms during the postpartum period. DM in pregnancy did not delay the postpartum pelvic floor recovery, suggesting that in young women the main effect is of pregnancy itself, and that the effect of DM on the pelvic floor seen in other studies may be related to a long-standing disease. Future studies should focus on evaluating PFD stratified by the different types of DM during pregnancy (gestational vs pregestational) and according to diabetic control status as well as evaluate the long-term effects of DM in pregnancy on PFD.

\section{Contributions}

Concept: N.B.T., M.E., D.Y., A.Y.W., Design: N.B.T., M.E., D.Y., A.Y.W., Data Collection or Processing: N.B.T., M.E., Z.Y., H.G., E.P., D.T., Analysis or Interpretation: N.B.T., Y.B., Z.Y., D.T., D.Y., A.Y.W., Literature Search: N.B.T., D.T., A.Y.W., Writing: N.B.T., Y.B., D.Y., A.Y.W.

\section{Ethics}

Ethics Committee Approval: Approval of the Institutional Review Board was obtained (Department of Obstetrics and Gynecology at the Soroka University Medical Center (SUMC), Beer Sheva, Israel) (IRB decision number: SOR-199-16, approval date: 07/07/2016).

Informed Consent: All women gave their consent for a follow-up questionnaire three months after delivery, to reflect the women's state during the postpartum period.

Peer-review: Externally peer-reviewed.

\section{DISCLOSURES}

Conflict of Interest: The authors declare that they have no conflict of interest.

Financial Disclosure: This research did not receive any specific grant from funding agencies in the public, commercial, or notfor-profit sectors.

\section{REFERENCES}

1. Bovbjerg VE, Trowbridge ER, Barber MD, Martirosian TE, Steers WD, Hullfish KL. Patient-centered treatment goals for pelvic floor disorders: association with quality-of-life and patient satisfaction. Am J Obstet Gynecol 2009; 200: 568.e1-6.

2. Black KI, Fraser IS. The burden of health associated with benign gynecological disorders in low-resource settings. Int J Gynaecol Obstet 2012; 119: 72-75.

3. Burgio KL, Borello-France D, Richter HE, et al. Risk factors for fecal and urinary incontinence after childbirth: the childbirth and pelvic symptoms study. Am J Gastroenterol 2007; 102: 1998-2004.
4. Boyles SH, Li H, Mori T, Osterweil P, Guise JM. Effect of mode of delivery on the incidence of urinary incontinence in primiparous women. Obstet Gynecol 2009; 113: 134-141.

5. Huebner M, Antolic A, Tunn R. The impact of pregnancy and vaginal delivery on urinary incontinence. Int J Gynaecol Obstet 2010; 110: 249-251.

6. Brown SJ, Gartland D, Donath S, MacArthur C. Effects of prolonged second stage, method of birth, timing of caesarean section and other obstetric risk factors on postnatal urinary incontinence: an Australian nulliparous cohort study. BJOG 2011; 118: 991-1000.

7. Moyer VA; U.S. Preventive Services Task Force. Screening for gestational diabetes mellitus: U.S. Preventive Services Task Force recommendation statement. Ann Intern Med 2014; 160: 414-420.

8. O'Neill S, O'Driscoll L. Metabolic syndrome: a closer look at the growing epidemic and its associated pathologies. Obes Rev 2015; 16: $1-12$.

9. Langer O, Yogev Y, Most O, Xenakis EM. Gestational diabetes: the consequences of not treating. Am J Obstet Gynecol 2005; 192: 989997.

10. Brown JS, Vittinghoff E, Lin F, Nyberg LM, Kusek JW, Kanaya AM. Prevalence and risk factors for urinary incontinence in women with type 2 diabetes and impaired fasting glucose: findings from the National Health and Nutrition Examination Survey (NHANES) 20012002. Diabetes Care 2006; 29: 1307-1312.

11. Chuang CM, Lin IF, Horng HC, Hsiao YH, Shyu IL, Chou P. The impact of gestational diabetes mellitus on postpartum urinary incontinence: a longitudinal cohort study on singleton pregnancies. BJOG 2012; 119: 1334-1343.

12. Barber MD, Kuchibhatla MN, Pieper CF, Bump RC. Psychometric evaluation of 2 comprehensive condition-specific quality of life instruments for women with pelvic floor disorders. Am J Obstet Gynecol 2001; 185: 1388-1395.

13. Barber MD, Chen Z, Lukacz E, et al. Further validation of the short form versions of the Pelvic Floor Distress Inventory (PFDI) and Pelvic Floor Impact Questionnaire (PFIQ). Neurourol Urodyn 2011; 30: 541-546.

14. Lowenstein L, Levy G, Chen KO, Ginath S, Condrea A, Padoa A. Validation of Hebrew versions of the Pelvic Floor Distress Inventory, Pelvic Organ Prolapse/Urinary Incontinence Sexual Function Questionnaire, and the Urgency, Severity and Impact Questionnaire. Female Pelvic Med Reconstr Surg. 2012; 18: 329-331.

15. Yohay D, Weintraub AY, Mauer-Perry N, et al. Prevalence and trends of pelvic floor disorders in late pregnancy and after delivery in a cohort of Israeli women using the PFDI-20. Eur J Obstet Gynecol Reprod Biol 2016; 200: 35-39.

16. Barber MD, Walters MD, Bump RC. Short forms of two conditionspecific quality-of-life questionnaires for women with pelvic floor disorders (PFDI-20 and PFIQ-7). Am J Obstet Gynecol 2005; 193: $103-$ 113.

17. Wong T, Ross GP, Jalaludin BB, Flack JR. The clinical significance of overt diabetes in pregnancy. Diabet Med 2013; 30: 468-474. 
18. Wesnes SL, Hunskaar S, Bo K, Rortveit G. The effect of urinary incontinence status during pregnancy and delivery mode on incontinence postpartum. A cohort study. BJOG 2009; 116: 700-707.

19. Handa VL, Zyczynski HM, Burgio KL, et al. The impact of fecal and urinary incontinence on quality of life 6 months after childbirth. Am J Obstet Gynecol 2007; 197: 636.e1-6.

20. Sangsawang B. Risk factors for the development of stress urinary incontinence during pregnancy in primigravidae: a review of the literature. Eur J Obstet Gynecol Reprod Biol 2014; 178: 27-34.

21. Harvey MA, Johnston SL, Davies GA. Mid-trimester serum relaxin concentrations and post-partum pelvic floor dysfunction. Acta Obstet Gynecol Scand 2008; 87: 1315-1321.

22. Ebbesen MH, Hannestad YS, Midthjell K, Hunskaar S. Diabetes and urinary incontinence - prevalence data from Norway. Acta Obstet Gynecol Scand. 2007; 86: 1256-1262.

23. Lawrence JM, Lukacz ES, Liu IL, Nager CW, Luber KM. Pelvic floor disorders, diabetes, and obesity in women: findings from the
Kaiser Permanente Continence Associated Risk Epidemiology Study. Diabetes Care 2007; 30: 2536-2541.

24. Brown JS, Nyberg LM, Kusek JW, et al. Proceedings of the National Institute of Diabetes and Digestive and Kidney Diseases International Symposium on Epidemiologic Issues in Urinary Incontinence in Women. Am J Obstet Gynecol. 2003; 188: 77-88.

25. Baltzis D, Eleftheriadou I, Veves A. Pathogenesis and treatment of impaired wound healing in diabetes mellitus: new insights. Adv Ther 2014; 31: 817-836

26. Micussi MT, Freitas RP, Angelo PH, Soares EM, Lemos TM, Maranhão TM. Evaluation of the relationship between the pelvic floor muscles and insulin resistance. Diabetes Metab Syndr Obes 2015; 8: 409-413.

27. Kim C, McEwen LN, Sarma AV, Piette JD, Herman WH. Stress urinary incontinence in women with a history of gestational diabetes mellitus. J Womens Health (Larchmt) 2008; 17: 783-792. 